\title{
Modelo de negocios Canvas: análisis de sus horizontes epistemológicos
}

\section{Canvas business model: analysis of their epistemological horizons}

Juan Carlos Carriel Wang

Steve Freddy Guarnizo Crespo

Luis Asencio Cristobal

Universidad de Guayaquil, Ecuador

Autor para correspondencia: juan.carrielw@ug.edu.ec, steve.guarnizoc@ug.edu.ec, Luis.asencio@ug.edu.ec

Fecha de recepción: 4 de enero de 2017 - Fecha de aceptación: 20 de Febrero de 2017

Resumen: El objeto de la investigación es analizar los componentes del modelos de negocios Canvas para deducir su horizonte epistemológico y contrastarlo con el paradigma del modelo educativo de Universidad de Guayaquil, Facultad de Ciencias Administrativas, tipología de trabajo de titulación : Modelos de Negocio. El alcance del estudio es exploratorio y descriptivo, se utilizó el método teórico de análisis- síntesis y el método empírico a través de encuestas; dirigidas a estudiantes del último nivel, entrevistas a docentes tutores y expertos. Se reflexiona y debate que el modelo de negocio Canvas, contiene un enfoque epistemológico de carácter tecnológico o conectivismo desde lo empírico, positivismo, mercantilismo, que no evoluciona a lo holístico y critico social; por tanto se prioriza: el mercado- empresa-inversión financiera y no a la sociedad que la acoge, coincide con los componentes del modelo de negocio para trabajos de titulación. No, así la Universidad de Guayaquil quien proclama un modelo educativo por competencias y procesos, de visión holístico y crítico social, sistémico. Se delega para futuras investigaciones de carácter correlacional y explicativa; el diseño de un modelo de negocios de factibilidad económico social que determine la visión económica- social de la universidad y su relación formativa en futuros graduados.

Palabras Claves: modelos de negocios canvas; horizontes epistemológicos; universidad de guayaquil

\begin{abstract}
The object of the research is to test the relationships of the components of business models "Canvas " with studies of economic and social feasibility analyze their epistemological horizon from the type of work titling Business Models. The scope of the study is exploratory and descriptive, it was used the theoretical analysis- synthesis method and empirical method through surveys directed students In this paper is torn, and reflects the business model Canvas, it contains an epistemological approach empirical, positivist and pragmatic character that evolves not social; therefore it prioritizes such as: the market- business - financial investment and not to the society that welcomes them. Preventing future research correlational and explanatory character; designing a business model social economic feasibility economically determined social vision and entrepreneurship of future professionals MKT \& NC. Likewise, the University of Guayaquil (UG), proclaims an educational model for critical social, systemic competencies; where the training of its professionals through new curricula; first openly express requirement to contribute to society as a whole; Construction of the production model, the gender and other positive externalities vulnerable and strategic sector.
\end{abstract}

Key words: canvas business models; epistemological horizon; guayaquil university 


\section{Introducción}

El perfil de egreso de un estudiante de ciencias administrativas de la Universidad de Guayaquil (UG); manifiestas entre sus enunciados: El egresado de la Facultad De Ciencias Administrativas (Fca-ug) está capacitado para presentar proyectos con compromiso social a las demandas del entorno y protección del medio ambiente. Elaborar técnicamente informes de las investigaciones del mercado. Elaborar planes estratégicos y proyectos de acuerdo a la realidad socio-económica. Actuar con responsabilidad en la segmentación del mercado. Aplicar con honestidad principios éticos y morales en el desempeño de sus funciones.

La Fca-ug a través de la unidad de titulación adopto por resolución del consejo académico, para el periodo 2016-II entre sus tipologías de trabajo de titulación el modelo de Negocios, cuyo protocolo o componentes se teoriza semejanzas con el modelo de negocio Canvas, el mismo tiene un enfoque mercantilista y relación mercado, finanzas, ante ello se plantea el interrogante : ¿A quién deben de servir los futuros profesionales formados en el alma mater a un sistema empresarial o la sociedad en todo su conjunto? Sin duda a la sociedad en todo su conjunto, lo cual es necesario de contestar y aún más de aplicar

\subsection{Objetivo}

El propósito del ensayo; pretende comparar teóricamente los componentes del modelo de negocio Canvas con modelos de factibilidad económica social, para analizar su horizonte epistemológico y debatir su pertinencia social al perfil de egreso y modelo educativo institucional de la Universidad de Guayaquil-Fca-ug.

\subsection{Justificación}

En los nuevos diseños curriculares se discute un eje transversal para todas las asignaturas en las diferentes mallas y diferentes carreras, al modelo de educación adquirido y proclamado por la Universidad de Guayaquil UG, esto es el Critico Social, que en su enfoque ecológico pluralista y de diversidad epistemológico da la lectura de su misión y visión.

Uno de los problemas que enfrenta el sistema de educación superior tiene que ver con las tasa de eficiencia terminal que se expresa en estudiantes que se titulan tardíamente o desertan de su formación profesional. Según datos del Senecyt "Solo 11 de cada 100 alumnos de universidades públicas se gradúan con su correspondiente cohorte, en el 2013 se constató que existen más de 60.000 estudiantes que habiendo concluido las asignaturas de la malla curricular, no han elaborado su trabajo de grado y por tanto no están titulados" (Larrea 2014)

Por tanto un objetivo básico, para todas las unidades académicas de titulación de la Universidad de Guayaquil y del país, es el de mejorar dicho índice, así están en proceso de elección o ya han elegido diferentes tipologías de trabajos de graduación, entre ellas el modelo de negocio. Debiendo de caracterizarse el mismo con componentes técnicos y sociales de acuerdo al perfil profesional. El analizar los componentes del modelo de negocios Canvas y relacionarlos con el perfil de egreso de los estudiantes; enriquecerá la decisión de elección y recreará su horizonte epistemológico. 


\section{Marco Teórico}

Un modelo de negocio describe la lógica de como una organización, crea, entrega y captura valor, el valor no siempre es medido en dinero, pues el valor de las cosas pueden ser funcionales o emocionales.

En la actualidad, la era de la globalización, conocimiento y de las tecnologías de la información y la comunicación Tics, las empresas e instituciones a través de sus directivos, gerentes y colaboradores buscan diferentes estrategias para mantener o generar una ventaja competitiva que permita estar a la vanguardia o mantener su cota de mercado, y esa oportunidad es diseñada utilizando modelos de negocios y de ahí la importancia de innovar y generar actualizaciones del mismo.

Según Osterwalder (2011). La revolución tecnológica de las últimas dos décadas. Internet, engendró una nueva economía y un nuevo tipo de empresas: las startup, una startup realmente está diseñada para buscar rentabilidad; que la inversión genere utilidades, de lo contrario es ir a la quiebra; además que todo los procesos tengan algo en común, repetible, encontrar procesos de ventas, mercadeo e ingeniería que sean medibles, escalable, como es repetible se empieza la búsqueda del modelo de negocio considerado la manera o método en que una empresa crea valor para sí misma al mismo tiempo que entrega productos o servicios a sus clientes .

Al comparar los modelos de negocios con un análisis de factibilidad económico social, el concepto de modelo factible ha tenido una evolución importante, desde lo económico financiero a lo social y complejo, inicialmente pertenecía básicamente al campo de la ingeniería, posteriormente se le agregan las estimaciones financieras de los costos e ingresos y hoy es un campo multidisciplinario profesionalmente, lo cual le da un criterio inter-disciplinar, holista sistémico.

Ernesto Fontainne (2006) describe para "un Economista, es la fuente de costos y beneficios, que ocurren en diferentes periodos de tiempo; para un Administrador de Empresas es una ordenación de actividades y recursos que se realizan con el fin de producir bienes o servicios". (Fontainne, 2006, p. 21).

Si bien las aprensiones empírica y positivista de Ernesto Fontainne, señala los sesgos conceptuales aislados entre los enfoques economicistas y del administrador de empresas; este autor propone incorporar la orientación de otras disciplinas con enfoque critico social, hermenéutico, que deben ser conjugados en aras de conseguir un equipo de trabajo, que formule y evalué, las opciones técnicas y tecnológicas en las diferentes ramas especializadas, así ingeniero de las diferentes especialidades: ambientales, industriales, civiles, químicos, de alimentos, contadores, sociólogos, psicólogos, publicistas, abogados, técnicos y más trabajadores especializados, que logre los mayores éxitos en un proyecto de inversión.

En el mismo ámbito, Nassir Sapag (2012), incorpora al individuo al resultado del modelo, pues a pesar que el mismo surge como repuesta a una idea que busca ya sea la solución de un problema o la forma de aprovechar una oportunidad de negocio, está propuesto a conseguir un 
mejor estándar de vida a sus beneficiarios. De igual forma Karen Mokate (2011), hace referencia que el modelo de negocios debe servir al mejoramiento de los actores, incorporando a todos los grupos beneficiarios e involucrados.

La Norma de calidad ISO 10006 describe al modelo de negocios como un proceso único, que consiste de un conjunto de actividades coordinadas y controladas, con fecha de inicio y término que son emprendidas para alcanzar un objetivo, incluyendo restricciones de plazo, costos y recursos.

Además del enfoque de administración de procesos, expuesto en las Normas ISO, en términos propios se concibe a un modelo de negocio, como un vía en espiral, que busca cambiar una situación real, por lo general no deseada, a una situación mejorada, para la cual se utilizan un conjunto de estudios que se interrelacionan entre sí, para una vez evaluados sirvan de apoyo a la toma de decisión de una inversión.

Las actualizaciones del valor de los modelos de negocios continúan con visión positivista e empírica, monetarista, mercadotecnita en: segmentos de mercado, creación de valor, canales de distribución, relaciones con los clientes, fuentes de ingresos, recursos, actividades, asociaciones, estructura de costos, íntegramente se busca satisfacer las necesidades del cliente, lo que es diferente a satisfacer las necesidades de la sociedad con visión de crítica social. Al analizar el modelo de negocio Fca-ug en el protocolo de resumen ejecutivo, se encuentra entre sus apartados del modelo: Es la descripción de valor que una empresa ofrece a uno o varios segmentos de clientes y de la arquitectura de la empresa y su red de socios para crear, comercializar y aportar este valor, a la vez que genera un flujo rentable y sostenible de ingresos.

"Valor Potencial del mercado, entorno y factibilidad comercial del proyecto, propuesta de valor del producto /servicio, ¿porque alguien tiene que comprar mi producto/servicio? Diferenciación, porque soy una opción diferente, nueva, mejor, liderazgo destacar experiencia y capacidades relevantes de líderes. Información financiera relevante: inversión, pronóstico de ventas del primer año, costos y gastos, período de recuperación de la inversión, rentabilidad sobre la Inversión y sobre el capital contable" (UG; FCA; Modelo de Negocio MK\&NC; 2016). Para el método teórico de análisis- síntesis se consolida la matriz de Análisis comparativo de los componentes del modelo de negocio.

Tabla 1. Matriz de análisis comparativo de los componentes del modelo de negocios

\begin{tabular}{lll}
\hline $\begin{array}{l}\text { Componentes } \\
\text { del modelo }\end{array}$ & Modelo de Negocios Canvas & Modelos De Factibilidad Económico Y Social \\
\hline \multirow{2}{*}{ Mercado } & $\begin{array}{l}\text { Necesidades del cliente, oferta demanda, } \\
\text { competencia, marketing Mix, slogan, } \\
\text { logotipo distribución, estrategias de } \\
\text { comercialización }\end{array}$ & $\begin{array}{l}\text { Necesidades de la sociedad, equidad, eficiencia, } \\
\text { oferta y demanda social, precios sombras, } \\
\text { mercadotécnica social }\end{array}$ \\
\hline & $\begin{array}{l}\text { Relación Mercado-maquina-hombre- } \\
\text { equipos-tecnología, tamaño y } \\
\text { localización por mercado, procesos, } \\
\text { control de calidad, medio ambiente }\end{array}$ & $\begin{array}{l}\text { Relación Matriz Productiva, ergonomía, tamaño } \\
\text { yroducalización por sectores vulnerables, calidad } \\
\text { ecolónica }\end{array}$ \\
\hline $\begin{array}{l}\text { Estructura organizacional, jerarquía, } \\
\text { Humanos }\end{array}$ & $\begin{array}{l}\text { competencias, manuales de funciones } \\
\text { Capital humano, Identidad de género, } \\
\text { capacidades especiales, competencias, } \\
\text { funciones. }\end{array}$ \\
\hline
\end{tabular}




\begin{tabular}{lll}
\hline $\begin{array}{l}\text { Contabilidad y } \\
\text { Finanzas }\end{array}$ & $\begin{array}{l}\text { Rentabilidad, maximizar beneficios, } \\
\text { Indicadores financieros de inversión } \\
\text { (VAN, TIR, PRI) }\end{array}$ & $\begin{array}{l}\text { Maximizar Externalidades positivas a la } \\
\text { sociedad, costos evitados, eficiencia financiera } \\
\text { (Van Social, TIR, PRI) }\end{array}$ \\
\hline $\begin{array}{l}\text { Aspectos } \\
\text { legales }\end{array}$ & $\begin{array}{l}\text { Persona natural, jurídica, empresa } \\
\text { privada, }\end{array}$ & $\begin{array}{l}\text { Empresas de la economía popular y solidaria, ley } \\
\text { del consumidor y regulaciones de monopolio, } \\
\text { poder de mercado, inversión pública, privada, } \\
\text { asociatividad, persona natural, jurídica }\end{array}$ \\
\hline
\end{tabular}

\section{Materiales y métodos}

El alcance del estudio es cuantitativo y cualitativo, se utilizó el método teórico de análisis- síntesis y el método empírico a través de encuestas; dirigidas a estudiantes del último nivel, entrevistas a docentes tutores y expertos, se consideró una muestra por conveniencia esto es: al $100 \%$ de estudiante (23) que realizan sus trabajos de titulación en la tipología de modelos de negocio Fca-ug, 23 docentes tutores, y 7 profesionales expertos docentes y revisores de trabajos de titulación.

\section{Procesamiento y análisis}

\section{Conocimiento del Modelo de Negocio Canvas}

Se cuestiona el conocimiento del modelo Canvas a estudiantes, docentes, y expertos; un gran porcentaje manifiesta tener conocimientos de la técnica del mismo.

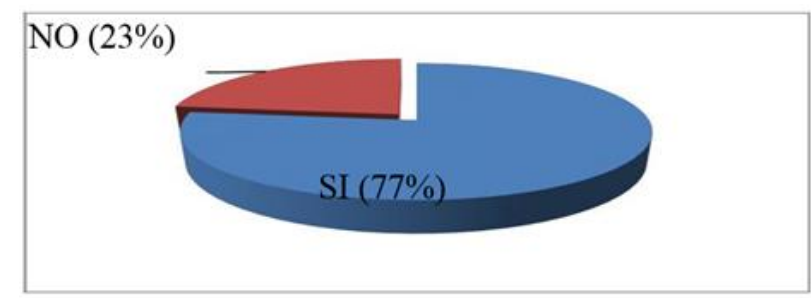

Figura 1.

\section{Similitud del modelo Canvas y modelo de negocio Fca-ug}

Entre los expertos, docentes y estudiantes investigados, manifiestan que existe un gran porcentaje de similitud entre los componentes del Modelo de negocio Canvas y el modelo de negocio Fca-ug

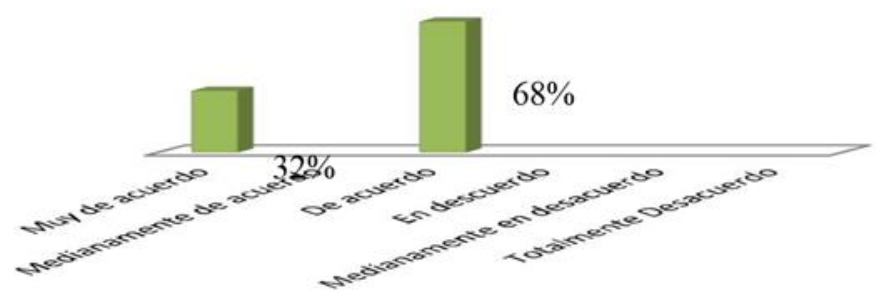

Figura 2. 


\section{Competencia y habilidades en estudiantes}

Las personas investigadas manifestaron que entre los enfoques de los componentes del modelo de negocio Canvas, prevalece los criterios mercadotecnita, de producción y financieros, siendo escasos los lineamientos sociales.

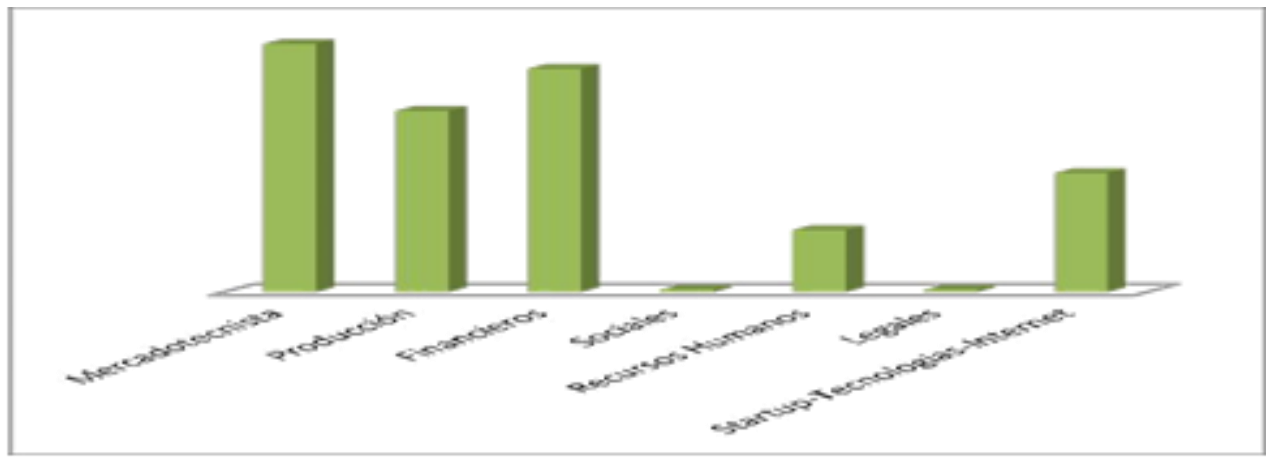

Figura 3. Competencia y habilidades en estudiantes Fca-ug, 2016

\section{Ranking de enfoques a los componentes del Modelo de negocio MKT\&NC}

Un gran porcentaje de docentes y expertos manifiestan que existe ausencia del paradigma crítico social, no así del positivismo, pragmatismo y conectivismo o (TIC’s) tecnologías de información y comunicación

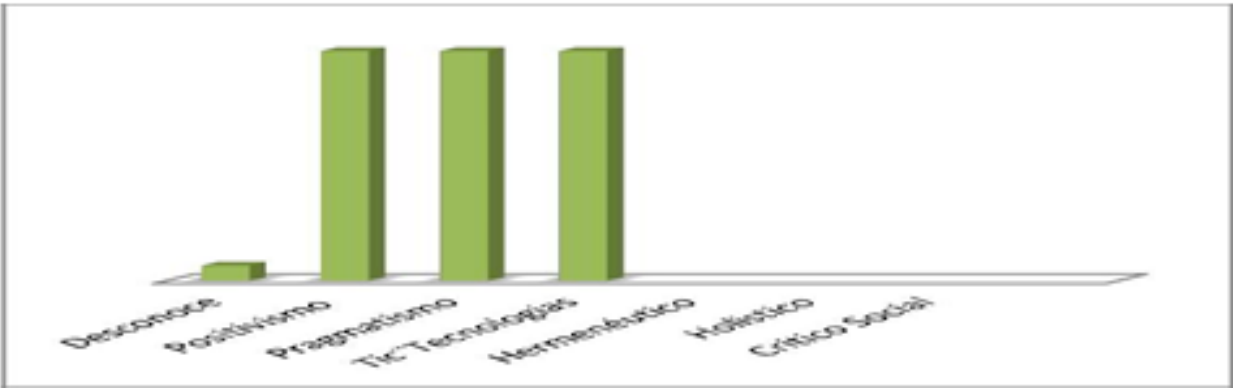

Figura 4. Paradigma epistemológico del modelo de negocio Fca-Ug, 2016

\section{Ranking de aplicación del modelo de negocio}

La aplicabilidad del modelo se alinea en gran porcentaje a la inversión privada, y en poca aplicabilidad a las estrategias de asociatividad y economía popular y solidaria y anula la acción social.

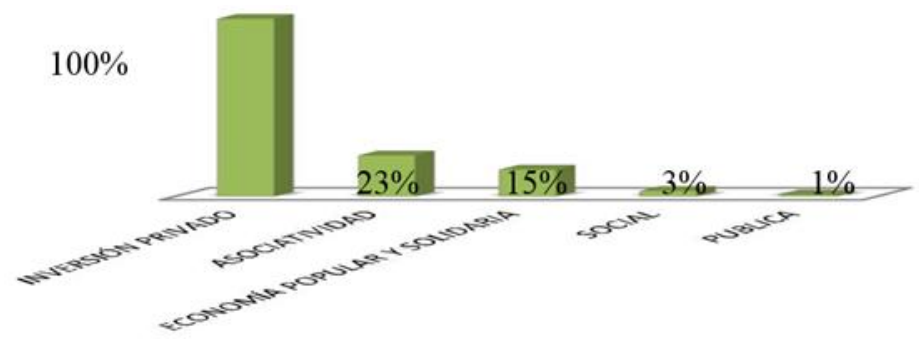

Figura 5. 


\section{Aportación al desarrollo profesional}

El modelo aporta al desarrollo profesional del Ingeniero en Ciencias administrativas: Emprender negocios propios utilizando ventajosamente negociaciones nacionales e internacionales vía On-line. Utilizar con creatividad e innovación técnicas publicitarias. Innovar acertadamente estrategias de marketing en el mercado. Establecer consistentemente ofertas de negocios en el mercado meta.

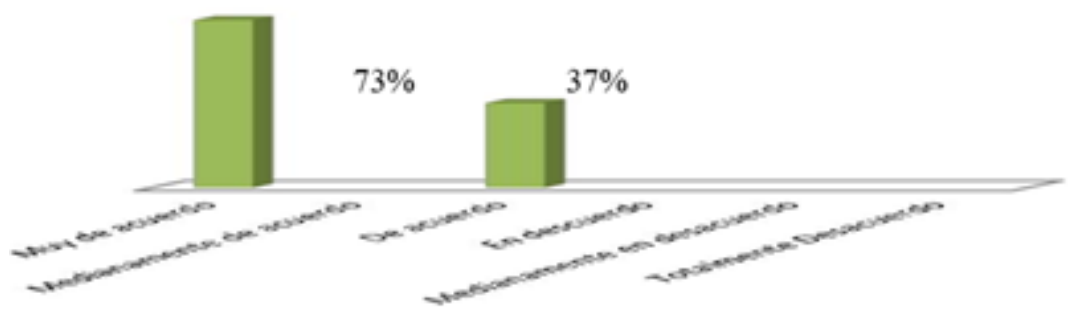

Figura 6. Aportación al desarrollo profesional Fca-ug, 2016

\section{Aporte social del modelo Fca-ug}

Los expertos y docentes entrevistados manifiestan el poco aporte social del modelo.

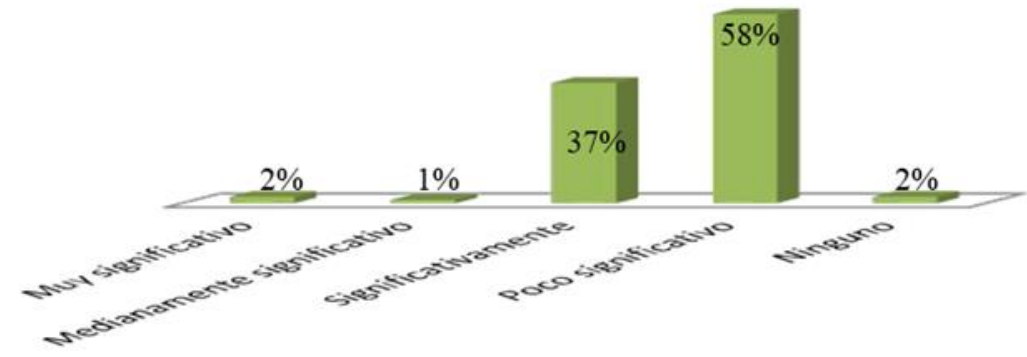

Figura 7. Aporte social del modelo Fca-ug al modelo educativo social-ecológico, 2016

\section{Debate y discusión}

Se debate, y reflexiona: La evaluación de negocios e inversiones temporales con un enfoque economicista, financiero, mercadotecnita que valora la riqueza y generación de la misma en relación al dinero y su complicada evolución a la evaluación de las inversiones con enfoque económico social resulta cada vez más importante y necesaria en el mundo contemporáneo.

En el Ecuador esta necesidad cobra mayor significación, dado el insuficiente desarrollo económico social, este enfoque es tanto más apremiante en la medida que el componente mercantil está presente con su sesgo de racionalidad microeconómica, que debe ser incluida convenientemente en aras de los intereses sociales. La dicotomía planificación - mercado equivale a la del interés social - interés empresarial o de grupo. El modelo de negocio Canvas y sus componentes de mercado, producción y finanzas, poseen un gran porcentaje de similitud con el modelo de negocios adoptado por la Facultad de Ciencias Administrativas en la Universidad de Guayaquil. 
El modelo de negocio para la tipología de trabajos de titulación de la Facultad de Ciencias Administrativas, Universidad de Guayaquil, por ahora asume un horizonte epistemológico posicionado en el positivismo pragmático y conectivismo, carece de carácter critico social, manteniendo un enfoque monetarista y mercadotecnita que se aleja del modelo educativo de la universidad en general.

La solución de dicha contradicción, se aguarda para futuras investigaciones de carácter correlacional y explicativa; al diseño de un modelo de negocios de factibilidad económico social que determine la visión económica- social de los futuros graduados Fca-ug, y emprendimientos. La cual encuentra en el enfoque social de la evaluación de negocios e inversiones, una fórmula de conciliación positivista, pragmática, de crítica social, sistemática y compleja, utilizando las TIC".s en la prosperidad benéfica.

Los conceptos teóricos del modelo de negocio que se indica como modelo factible; incluyendo constituir e interrelacionar aspectos a debatir, como:

- Una lectura de la realidad social, que evidencia que ésta, o alguno de sus aspectos, deben ser objeto de evolución.

- Un enfoque para generar externalidades sociales que mejoren el estándar de vida de la sociedad, disminuyan la brecha de pobreza de los ciudadanos, generando riqueza y equidad.

\section{Bibliografía}

Proyecto de unidad de titulación, universidad de Guayaquil, Facultad de Ciencias Administrativas, carrera de Ingeniería en Marketing Y negociación Comercial., Zambrano Mario \& Coello Viviana, 2015.

Generación de modelos de Negocios, Alexander Osterwilder \& Yves Pigneur. Bisness Model Generation.Com, 2011.

MÉNDEZ Carlos, Metodología. Guía para elaborar diseños de investigación en ciencias económicas, contables y administrativas, Bogotá, Editorial McGraw

- Hill Interamericana S.A., Segunda Edición, 2010.

MOKATE Karen, Formulación y Evaluación de proyectos de inversión y sociales, 2010.

SAPAG José, Evaluación de proyectos guía de ejercicios problema y soluciones, Editorial McGraw - Hill Interamericana, S.A., 2011

SAPAG Nassir y Sapag Reynaldo, Preparación y evaluación de proyectos, Editorial McGraw Hill Interamericana, S.A., 2011. 